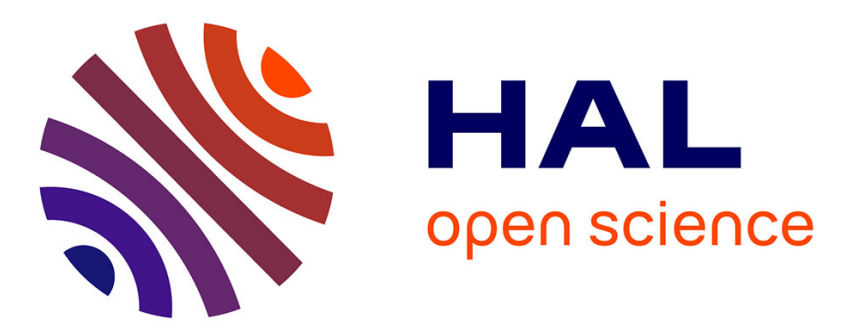

\title{
A non-linear symmetry-preserving observer for velocity-aided inertial navigation
}

Silvère Bonnabel, Philippe Martin, Pierre Rouchon

\section{To cite this version:}

Silvère Bonnabel, Philippe Martin, Pierre Rouchon. A non-linear symmetry-preserving observer for velocity-aided inertial navigation. 2006 American Control Conference, Jun 2006, France. pp.ThB03.6, 10.1109/ACC.2006.1657161 . hal-00533355

\section{HAL Id: hal-00533355}

https://hal-mines-paristech.archives-ouvertes.fr/hal-00533355

Submitted on 5 Nov 2010

HAL is a multi-disciplinary open access archive for the deposit and dissemination of scientific research documents, whether they are published or not. The documents may come from teaching and research institutions in France or abroad, or from public or private research centers.
L'archive ouverte pluridisciplinaire HAL, est destinée au dépôt et à la diffusion de documents scientifiques de niveau recherche, publiés ou non, émanant des établissements d'enseignement et de recherche français ou étrangers, des laboratoires publics ou privés. 


\title{
A non-linear symmetry-preserving observer for velocity-aided inertial navigation
}

\author{
Silvere Bonnabel, Philippe Martin and Pierre Rouchon
}

\begin{abstract}
A first theory of invariant observers is developed. An invariant observer is an observer which respects the symmetries of the system equations. As an illustration of the theory, a nonlinear invariant observer for velocity-aided inertial navigation is proposed and analyzed.
\end{abstract}

The operation of an aircraft, whether manual, computerassisted or fully automatic, requires a good knowledge of (at least) its velocity vector and orientation. In low-cost systems navigation systems, the (relatively) inaccurate gyroscopes and accelerometers are "aided" by velocity measurements (given by an air-data system or a Doppler radar). The various measurements are then "merged" according to the (flat-Earth) motion equations of the aircraft, usually by a gain-scheduled observer (or in a more sophisticated way by an extended Kalman filter). The convergence analysis, hence the tuning, of such an observer is far from easy.

In this paper, we propose as an alternative a simple nonlinear observer. It is designed so as to respect the natural symmetries of the motion equations, which are derived from galilean invariance. It has a strong differential geometric structure, which yields a local convergence property around any trajectory of the aircraft. Symmetries have been used in control theory for feedback design and optimal control, see for instance [3], [4], [8], [9], [5], [10] but seemingly much less for observer design [2], [1]. This paper also proposes a theory for invariant observers extending the ideas and results of [1]; it can be seen as the counterpart for observer design of [5] for invariant tracking controller design.

The paper is organized as follows: the velocity-aided inertial navigation problem is precisely stated in section I; in section II the nonlinear invariant observer is described and analyzed; section III is devoted to a general theory of invariant pre-observers; this theory is used in section IV to derive the observer of section II.

\section{VELOCITY-AIDED INERTIAL NAVIGATION}

\section{A. Motion equations}

The motion of a flying rigid body (assuming the Earth is flat and defines an inertial frame) is described by

$$
\begin{aligned}
& \dot{q}=\frac{1}{2} q * \vec{\omega} \\
& \dot{\vec{v}}=\vec{v} \times \vec{\omega}+q^{-1} * \vec{g} * q+\vec{a},
\end{aligned}
$$

where $q$ is the quaternion representing the orientation of the body-fixed frame with respect to the Earth-fixed frame, $\vec{\omega}$ is

S. Bonnabel, Ph. Martin and P. Rouchon are with Centre Automatique et Systèmes, École des Mines de Paris, 60 boulevard Saint-Michel, 75272 Paris CEDEX 06, FRANCE silvere.bonnabeldensmp.fr, philippe.martin@ensmp. fr, pierre.rouchondensmp. the instantaneous angular velocity vector, $\vec{v}$ is the velocity vector of the center of mass with respect to a body-fixed frame, $\vec{g}$ is the (constant) gravity vector, $\vec{a}$ is the specific acceleration vector, and represents here the aerodynamics forces divided by the body mass.

The first equation describes the kinematics of the body, the second is Newton's force law. It is customary to use quaternions (also called Euler 4-parameters) instead of Euler angles since they provide a global parametrization of the body orientation, and are well-suited for calculations and computer simulations.

One can briefly recall a quaternion $p$ can be thought of as a scalar $p_{0} \in \mathbb{R}$ together with a vector $\vec{p} \in \mathbb{R}^{3}, p=\left(p_{0}, \vec{p}\right)$. The quaternion product $*$ then reads

$$
p * q:=\left(\begin{array}{c}
p_{0} q_{0}-\vec{p} \cdot \vec{q} \\
p_{0} \vec{q}+q_{0} \vec{p}+\vec{p} \times \vec{q}
\end{array}\right) .
$$

Thus any vector $\vec{p} \in \mathbb{R}^{3}$ can be seen as the quaternion $p:=(0, \vec{p})$. Moreover, to any quaternion $q$ with unit norm is associated a rotation matrix $R_{q} \in S O(3)$ by the following relation: $q^{-1} * \vec{p} * q=R_{q} \cdot \vec{p}$ for all $\vec{p}$.

\section{B. Velocity-aided inertial navigation}

The operation of an aircraft, whether manual, computerassisted or fully automatic, requires a good knowledge of (at least) its velocity vector and orientation. In "true" inertial navigation systems, very accurate -and very expensive- gyroscopes and accelerometers are used to measure the angular velocity vector $\vec{\omega}$ and the specific acceleration $\vec{a}$. In low-cost systems "strap-down" systems, the measurements (in bodyfixed axes) of $\vec{\omega}$ and $\vec{a}$ by (rather) cheap gyroscopes and accelerometers are complemented by the measurement (also in body-fixed axes) of the velocity vector $\vec{v}$ by an air-data system or a Doppler radar. To make the most of these sensors, the various measurements must be appropriately "merged" according to the motion equations. This is usually done by a gain-scheduled observer (or by an extended Kalman filter) of the form

$$
\begin{aligned}
& \dot{\hat{q}}=\frac{1}{2} \hat{q} * \vec{\omega}-L_{q}(\hat{q}, \hat{v}, \vec{a}, \vec{\omega}) \cdot(\hat{\vec{v}}-\vec{v}) \\
& \dot{\hat{v}}=\hat{\vec{v}} \times \vec{\omega}+\hat{q}^{-1} * \vec{g} * \hat{q}+\vec{a}-L_{\vec{v}}(\hat{q}, \hat{v}, \vec{a}, \vec{\omega}) \cdot(\hat{\vec{v}}-\vec{v}) .
\end{aligned}
$$

The matrix function gains $L_{q}$ and $L_{\vec{v}}$ must be properly tuned to ensure the convergence of $(\hat{q}, \hat{\vec{v}})$ to $(q, \vec{v})$, which is not an easy task, even locally around a slowly-varying trajectory of the aircraft.

Notice that without an extra sensor there is one nonobservable degree of freedom: indeed, it is readily seen that 
only $\vec{v}$ and $q^{-1} * \vec{v} * q$ can be recovered from the knowledge of $\vec{v}, \vec{\omega}, \vec{a}$ and their derivatives, hence $q$ can be known only up to a rotation around $\vec{g}$. The extra (usually magnetic) sensor must be combined with the other sensors, which we do not address here for the sake of simplicity.

\section{Invariance of the motion equations}

The motion equations of a rigid body have a very specific geometry: they remain the same after performing any constant rotation of the body-fixed axes and any constant rotation around $\vec{g}$. To see this, consider the (invertible) transformation

$$
\left(\begin{array}{c}
Q \\
\vec{V} \\
\vec{A} \\
\vec{\Omega}
\end{array}\right):=\left(\begin{array}{c}
p * q * r \\
r^{-1} * \vec{v} * r \\
r^{-1} * \vec{a} * r \\
r^{-1} * \vec{\omega} * r
\end{array}\right)
$$

where $p$ is a quaternion such that $p^{-1} * \vec{g} * p=\vec{g}$, and $r$ is any quaternion. The transformed system reads

$$
\begin{aligned}
\dot{Q} & =p * \dot{q} * r \\
& =\frac{1}{2} p * q * r * r^{-1} * \vec{\omega} * r=\frac{1}{2} Q * \vec{\Omega} \\
\dot{\vec{V}} & =r^{-1} * \dot{\vec{v}} * r \\
& =r^{-1} *(\vec{v} \times \vec{\omega}) * r+r^{-1} * q^{-1} * \vec{g} * q * r+r^{-1} * \vec{a} * r \\
& =\left(r^{-1} * \vec{v} * r\right) \times\left(r^{-1} * \vec{\omega} * r\right)+(p * q * r)^{-1} * \vec{g} * p * q * r+\vec{A} \\
& =\vec{V} \times \vec{\Omega}+Q^{-1} * \vec{g} * Q+\vec{A},
\end{aligned}
$$

and is the same as the original one. In other words, the system is invariant by the transformation.

\section{A NONLINEAR INVARIANT OBSERVER}

A gain-scheduled observers (or more generally an extended Kalman filter) is very "linear" by design: its corrective terms are linear combinations of the "linear" output error $\hat{\vec{v}}-\vec{v}$ with arbitrary function coefficients, irrespective of the system geometry. This may account for the difficulty of analyzing its convergence. The following observer, geometrically much more "natural" will yield a very simple error system, hence convergence analysis.

\section{A. The observer}

Consider the observer

$$
\begin{aligned}
& \dot{\hat{q}}=\frac{1}{2} \hat{q} * \vec{\omega}-\hat{q} *\left[l_{1} \Delta \vec{v}+l_{2} \Delta \vec{v} \times \hat{\vec{G}}+l_{3}(\Delta \vec{v} \cdot \hat{\vec{G}}) \hat{\vec{G}}\right] \\
& \dot{\overrightarrow{\vec{v}}}=\hat{\vec{v}} \times \vec{\omega}+\hat{\vec{G}}+\vec{a}-\left[l_{4} \Delta \vec{v}+l_{5} \Delta \vec{v} \times \hat{\vec{G}}+l_{6}(\Delta \vec{v} \cdot \hat{\vec{G}}) \hat{\vec{G}}\right]
\end{aligned}
$$

where $l_{1}, \ldots, l_{6}$ are constant gains yet to be chosen and $\Delta \vec{v}:=$ $\hat{\vec{v}}-\vec{v}$ and $G:=\hat{q}^{-1} * \vec{g} * \hat{q}$. It can be seen as a gain-scheduled observer with geometry-preserving function gains. Indeed, consider the transformation (1) with $\hat{Q}=p * \hat{q} * r$ and $\hat{\vec{V}}=$ $r^{-1} * \hat{\vec{v}} * r$. In the capital-size variables, the observer dynamics reads

$$
\begin{aligned}
\dot{\hat{Q}} & =p * \frac{1}{2} \hat{q} * \vec{\omega} * r-p * \hat{q} *\left[l_{1} \Delta \vec{v}+l_{2} \Delta \vec{v} \times \hat{\vec{g}}+l_{3}(\Delta \vec{v} \cdot \hat{\vec{G}}) \hat{\vec{G}}\right] * r \\
& =p * \frac{1}{2} \hat{q} * r * r^{-1} * \vec{\omega} * r \\
& -p * \hat{q} * r *\left[l_{1} r^{-1} * \Delta \vec{v} * r+l_{2}\left(r^{-1} * \Delta \vec{v} * r\right) \times\left(r^{-1} * \hat{\vec{g}} * r\right)\right. \\
& \left.+l_{3}\left(\left(r^{-1} * \Delta \vec{v} * r\right) \cdot\left(r^{-1} * \hat{\vec{G}} * r\right)\right) r^{-1} * \hat{\vec{G}} * r\right] \\
& =\frac{1}{2} \hat{Q} * \vec{\Omega}-\hat{Q} *\left[l_{1} \Delta \vec{V}+l_{2} \Delta \vec{V} \times \hat{\vec{G}}+l_{3}(\Delta \vec{V} \cdot \hat{\vec{G}}) \hat{\vec{G}}\right]
\end{aligned}
$$

A similar computation gives

$$
\begin{aligned}
\dot{\overrightarrow{\vec{V}}} & =r^{-1} * \dot{\overrightarrow{\vec{V}}} * r \\
& =\hat{\vec{V}} \times \vec{\Omega}+\hat{\vec{G}}+\vec{A}-\left[l_{4} \Delta \vec{V}+l_{5} \Delta \vec{V} \times \hat{\vec{G}}+l_{6}(\Delta \vec{V} \cdot \hat{\vec{G}}) \hat{\vec{G}}\right]
\end{aligned}
$$

The differential equations of $\hat{Q}$ and $\hat{\vec{V}}$ remain identical to the ones of $\hat{q}$ and $\hat{\vec{v}}$.

\section{B. The error system}

Instead of considering the "linear" errors $\Delta q:=\hat{q}-q$ and $\Delta \vec{v}$, it will turn out to be much simpler to consider the (equivalent) errors

$$
\begin{aligned}
& e_{q}:=\Delta q * q^{-1} \\
& e_{\vec{v}}:=q * \Delta \vec{v} * q^{-1}
\end{aligned}
$$

$e_{q}$ is then the orientation error brought back around horizontal, and $e_{\vec{v}}$ is the velocity error brought back in Earth-fixed axes. Setting

$$
\begin{aligned}
O B S_{q}:=l_{1} \Delta \vec{v}+l_{2} \Delta \vec{v} \times \hat{\vec{G}}+l_{3}(\Delta \vec{v} \cdot \hat{\vec{G}}) \hat{\vec{G}} \\
O B S_{\vec{v}}:=l_{4} \Delta \vec{v}+l_{5} \Delta \vec{v} \times \hat{\vec{G}}+l_{6}(\Delta \vec{v} \cdot \hat{\vec{G}}) \hat{\vec{G}}
\end{aligned}
$$

we then find

$$
\begin{aligned}
\dot{e}_{q} & :=\Delta \dot{q} * q^{-1}+\Delta q *\left(-q^{-1} * \dot{q} * q^{-1}\right) \\
& =\left(\frac{1}{2} \Delta q * \vec{\omega}-\hat{q} * O B S_{q}-\frac{1}{2} \Delta q * \vec{\omega}\right) * q^{-1} \\
& =-\hat{q} * O B S_{q} * q^{-1} \\
\dot{e}_{\vec{v}} & :=\dot{q} * \Delta \vec{v} * q^{-1}-q * \Delta \vec{v} * q^{-1} * \dot{q} * q^{-1}+q * \Delta \dot{\vec{v}} * q^{-1} \\
& =q * \hat{\vec{G}} * q^{-1}-\vec{g}-q * O B S_{\vec{v}} * q^{-1},
\end{aligned}
$$

where

$\hat{\vec{g}}:=q * \hat{\vec{G}} * q^{-1}=q * \hat{q}^{-1} * \vec{g} * \hat{q} * q^{-1}=\left(1+e_{q}\right)^{-1} * \vec{g} *\left(1+e_{q}\right)$.

After computing the various terms in $O B S_{q}$ and $O B S_{\vec{v}}$,

$$
\begin{aligned}
q *(\Delta \vec{v} \times \hat{\vec{G}}) * q^{-1} & =\left(q * \Delta \vec{v} * q^{-1}\right) \times\left(q * \hat{\vec{G}} * q^{-1}\right) \\
& =e_{\vec{v}} \times \hat{\vec{g}} \\
q *(\Delta \vec{v} \cdot \hat{\vec{G}}) \hat{\vec{G}} * q^{-1} & =\left(e_{\vec{v}} \cdot \hat{\vec{g}}\right) \hat{\vec{g}} \\
\hat{q} *(\Delta \vec{v} \times \hat{\vec{G}}) * q^{-1} & =\left(1+e_{q}\right) *\left(e_{\vec{v}} \times \hat{\vec{g}}\right) \\
\hat{q} *(\Delta \vec{v} \cdot \hat{\vec{G}}) \hat{\vec{G}} * q^{-1} & =\left(1+e_{q}\right) *\left(e_{\vec{v}} \cdot \hat{\vec{g}}\right) \hat{\vec{g}},
\end{aligned}
$$


the error system eventually reads

$$
\begin{aligned}
& \dot{e}_{q}=-\left(1+e_{q}\right) *\left[l_{1} e_{\vec{v}}+l_{2} e_{\vec{v}} \times \hat{\vec{g}}+l_{3}\left(e_{\vec{v}} \cdot \hat{g}\right) \hat{g}\right] \\
& \dot{e}_{\vec{v}}=\hat{\vec{g}}-\vec{g}-\left[l_{4} e_{\vec{v}}+l_{5} e_{\vec{v}} \times \hat{\vec{g}}+l_{6}\left(e_{\vec{v}} \cdot \hat{\vec{g}}\right) \hat{\vec{g}}\right],
\end{aligned}
$$

with $\hat{\vec{g}}=\left(1+e_{q}\right)^{-1} * \vec{g} *\left(1+e_{q}\right)$.

A remarkable feature of the observer -very reminiscent of linear system theory- is that the resulting error system does not depend on $(q, \vec{v}, \vec{a}, \vec{\omega})$, as should be the case in general. In other words the observer has the same convergence whatever the trajectory of the flying body. This property makes the tuning of the observer gains very easy.

\section{Convergence of the linearized error system}

In this paper we will not analyze the stability of the nonlinear error system, but we will study the linearized error system around the equilibrium point $\left(\bar{e}_{\vec{v}}, \bar{e}_{q}\right):=(0,0)$. This linearized error system is readily seen to be

$$
\begin{aligned}
& \delta \dot{e}_{q}=-\left[l_{1} \delta e_{\vec{v}}+l_{2} \delta e_{\vec{v}} \times \vec{g}+l_{3}\left(\delta e_{\vec{v}} \cdot \vec{g}\right) \vec{g}\right] \\
& \delta \dot{e}_{\vec{v}}=2 \vec{g} \times e_{q}-\left[l_{4} \delta e_{\vec{v}}+l_{5} e_{\vec{v}} \times \vec{g}+l_{6}\left(\delta e_{\vec{v}} \cdot \vec{g}\right) \vec{g}\right],
\end{aligned}
$$

where we have used $\delta \hat{\vec{g}}=-\delta e_{q} * \vec{g}+\vec{g} * \delta e_{q}=2 \vec{g} \times e_{q}$.

In (Earth-fixed) coordinates, let

$$
\delta e_{q}:=\left(\begin{array}{c}
\delta e_{q}^{0} \\
-\delta e_{q}^{1} \\
\delta e_{q}^{2} \\
\delta e_{q}^{3}
\end{array}\right), \quad \delta e_{\vec{v}}:=\left(\begin{array}{c}
\delta e_{\vec{v}}^{1} \\
\delta e_{\vec{v}}^{2} \\
\delta e_{\vec{v}}^{3}
\end{array}\right) \quad \text { and } \quad \vec{g}=\left(\begin{array}{l}
0 \\
0 \\
g
\end{array}\right) \text {, }
$$

and the error system breaks into four decoupled subsystems: the horizontal subsystem

$$
\left(\begin{array}{c}
\delta \dot{e}_{\vec{v}}^{1} \\
\delta \dot{e}_{q}^{2} \\
\delta \dot{e}_{\vec{v}}^{2} \\
\delta \dot{e}_{q}^{1}
\end{array}\right)=\left(\begin{array}{cccc}
-l_{4} & -2 g & -g l_{5} & 0 \\
g l_{2} & 0 & -l_{1} & 0 \\
g l_{5} & 0 & -l_{4} & -2 g \\
l_{1} & 0 & g l_{2} & 0
\end{array}\right)\left(\begin{array}{c}
\delta e_{\vec{v}}^{1} \\
\delta e_{q}^{2} \\
\delta e_{\vec{v}}^{2} \\
\delta e_{q}^{1}
\end{array}\right)
$$

the vertical subsystem

$$
\delta \dot{e}_{\vec{v}}^{3}=-\left(l_{4}+g^{2} l_{6}\right) \delta \dot{e}_{\vec{v}}^{3}
$$

the heading subsystem, $\delta \dot{e}_{q}^{3}=-\left(l_{1}+g^{2} l_{3}\right) \delta \dot{e}_{\vec{v}}^{3}$, which is unobservable, and the quaternion norm subsystem, $\delta \dot{e}_{q}^{0}=0$.

The heading subsystem corresponds to the unobservable part (rotations around $\vec{g}$ ) and cannot be made convergent without an extra (e.g. magnetic) sensor.

The eigenvalue of the vertical subsystem can be freely assigned thanks to the gain $l_{6}$.

The four gains $l_{1}, l_{2}, l_{4}, l_{5}$ are used to assign the four of the longitudinal eigenvalues. They can be assigned completely freely because of the subsystem structure.

\section{ON INVARIANT OBSERVERS}

\section{A. Invariant systems and compatible outputs}

Definition 1: Let $G$ be a Lie Group with identity $e$ and $\Sigma$ an open set (or more generally a manifold). A transformation group $\left(\phi_{g}\right)_{g \in G}$ on $\Sigma$ is a smooth map

$$
(g, \xi) \in G \times \Sigma \mapsto \phi_{g}(\xi) \in \Sigma
$$

such that:

- $\phi_{e}(\xi)=\xi$ for all $\xi$

- $\phi_{g_{2}}\left(\phi_{g_{1}}(\xi)\right)=\phi_{g_{2} g_{1}}(\xi)$ for all $g_{1}, g_{2}, \xi$.

Notice $\phi_{g}$ is by construction a diffeomorphism on $\Sigma$ for all $g$. The transformation group is local if $\phi_{g}(\xi)$ is defined only when $g$ lies sufficiently near $e$. In this case the transformation law $\phi_{g_{2}}\left(\phi_{g_{1}}(\xi)\right)=\phi_{g_{2} g_{1}}(\xi)$ is imposed only when it makes sense. All the results of the paper being local, since based on constant rank assumptions, we consider in the sequel only local transformation groups acting on open sets. When we say "for all $g$ " we thus mean "for all $g$ sufficiently near the identity $e$ of $G$ "; in the same way "for all $\xi$ " usually means "for all generic $\xi$ in $\Sigma$ ". We use these shortcuts in order to improve readability.

Consider now the smooth output system

$$
\dot{x}=f(x, u) \quad y=h(x, u)
$$

where $x$ belongs to an open subset $\mathscr{X} \subset \mathbb{R}^{n}, u$ to an open subset $\mathscr{U} \subset \mathbb{R}^{m}$ and $y$ to an open subset $\mathscr{Y} \subset \mathbb{R}^{p}, p \leq n$.

We assume the signals $u(t), y(t)$ known ( $y$ is measured, and $u$ is measured or known as a control input).

Consider also the local group of transformations on $\mathscr{X} \times$ $\mathscr{U}$ defined by

$$
(X, U)=\left(\varphi_{g}(x), \psi_{g}(x, u)\right),
$$

where $\varphi_{g}$ is a local diffeomorphism and $\psi_{g}$ is invertible with respect to $u$ for all $x$ (which can be seen as a coordinate change and regular static state feedback).

Definition 2: The system $\dot{x}=f(x, u)$ is G-invariant if $f\left(\varphi_{g}(x), \psi_{g}(x, u)\right)=D \varphi_{g}(x) \cdot f(x, u)$ for all $g, x, u$.

The property also reads $\dot{X}=f(X, U)$, i.e., the system is left unchanged by the transformation.

Definition 3: The output $y=h(x, u)$ is G-compatible if there exists a transformation group $\left(\rho_{g}\right)_{g \in G}$ on $\mathscr{Y}$ such that $h\left(\varphi_{g}(x), \psi_{g}(x, u)\right)=\rho_{g}(h(x, u))$ for all $g, x, u$.

With $(X, U)=\left(\varphi_{g}(x), \psi_{g}(x, u)\right)$ and $Y=\rho_{g}(y)$, the definition means $Y=h(X, U)$.

\section{B. Invariant pre-observers}

Definition 4 (pre-observer): The system $\dot{\hat{x}}=F(\hat{x}, u, y)$ is a pre-observer of (2) if for all $x, u$

$$
F(x, u, h(x))=f(x, u) .
$$

This means every trajectory of the system is a trajectory of the pre-observer (the converse is false!); in other words $\hat{x}(0)=x(0)$ implies $\hat{x}(t)=x(t)$ for all $t \geq 0$.

Notice the definition says nothing about convergence; if moreover $\hat{x}(t) \rightarrow x(t)$ as $t \rightarrow+\infty$ for every (close) initial conditions, we say the pre-observer is asymptotic.

Example 1: the (linear) system

$$
\dot{\hat{x}}=F(\hat{x}, u, y):=A \hat{x}+B u-L(C \hat{x}+D u-y)
$$

is a pre-observer of the linear system

$$
\dot{x}=A x+B u \quad y=C x+D u
$$

since $F(x, u, C x+D u)=A x+B u$; it is an asymptotic observer when all the eigenvalues of $A-L C$ have negative real part. 
Definition 5: The pre-observer $\quad \dot{\hat{x}}=F(\hat{x}, u, y)$ is $\quad G$ invariant if for all $g, \hat{x}, u, y$,

$$
F\left(\varphi_{g}(\hat{x}), \psi_{g}(\hat{x}, u), \rho_{g}(y)\right)=D \varphi_{g}(\hat{x}) \cdot F(\hat{x}, u, y) .
$$

The property also reads $\dot{\hat{X}}=F(\hat{X}, U, Y)$, i.e., the system is left unchanged by the transformation.

\section{Characterization of invariant pre-observers}

In general the "usual" output error $\hat{y}-y=h(\hat{x}, u)-y$ does not preserve the system geometry, hence will not yield an invariant pre-observer. The key idea to build an invariant pre-observer is to use an invariant output error.

Definition 6: The smooth map $(\hat{x}, u, y) \mapsto E(\hat{x}, u, y) \in \mathbb{R}^{p}$ is an invariant output error if

- the map $y \mapsto E(\hat{x}, u, y)$ is invertible for all $\hat{x}, u$

- $E(\hat{x}, u, h(\hat{x}, u))=0$ for all $\hat{x}, u$

- $E\left(\varphi_{g}(\hat{x}), \psi_{g}(\hat{x}, u), \rho_{g}(y)\right)=E(\hat{x}, u, y)$ for all $\hat{x}, u, y$

The first and second properties mean $E$ is an "output error", i.e. it is zero if and only if $h(\hat{x}, u)=y$; the third property, which also reads $E(\hat{X}, U, Y)=E(\hat{x}, u, y)$, defines invariance.

The following theorem ensures the existence of a (local) invariant output error. The proof is constructive and relies on the Cartan moving frame method.

Theorem 1: Let $r$ be the dimension of the group $G$. Then

- there is an invariant output error $(\hat{x}, u, y) \mapsto E(\hat{x}, u, y)$

- there is a full-rank invariant map $(\hat{x}, u) \mapsto I(\hat{x}, u) \in$ $\mathbb{R}^{n+m-r}$ (i.e. a complete set of invariants)

- every other invariant output error reads

$$
\tilde{E}(\hat{x}, u, y)=\mathscr{L}(I(\hat{x}, u), E(\hat{x}, u, y)) .
$$

Proof: The result is an application of the moving frame method. We follow the nice presentation of [7, theorem 8.25]). Consider the transformation group $\left(\phi_{g}\right)_{g \in G}$ on $\Sigma \subset \mathbb{R}^{s}$ and assume $\partial_{g} \phi_{g}$ has full rank $r:=\operatorname{dim} G$ at the point $\left(e, \xi^{0}\right) \in G \times \Sigma$. We can then split $\phi_{g}$ into $\left(\phi_{g}^{a}, \phi_{g}^{b}\right)$ with respectively $r$ and $s-r$ components so that $\phi_{g}^{a}$ is invertible with respect to $g$ around $\left(e, \xi^{0}\right)$. The normalization equations are obtained by setting

$$
\phi_{g}^{a}(\xi)=c,
$$

with $c$ a constant in the range of $\phi$. The implicit function theorem ensures the existence of the local solution $g=\gamma(\xi)$ (the map $\gamma: \Sigma \rightarrow G$ is known as the moving frame). Finally, we get a complete set $J$ of $s-r$ functionally independent invariants by plugging $g=\gamma(\xi)$ into the remaining components,

$$
J(\xi):=\phi_{\gamma(\xi)}^{b}(\xi) .
$$

The invariance property means $J\left(\phi_{g}(\xi)\right)=J(\xi)$ for all $g$, $\xi$.

In our case $\Sigma=\mathscr{X} \times \mathscr{U} \times \mathscr{Y}$, and $\phi_{g}$ is the composite transformation

$$
\phi_{g}(\hat{x}, u, y):=\left(\varphi_{g}(\hat{x}), \psi_{g}(\hat{x}, u), \rho_{g}(y)\right) .
$$

Locally, we can split $(\hat{x}, u) \mapsto\left(\varphi_{g}(\hat{x}), \psi_{g}(\hat{x}, u)\right)$ into $\left(\varphi_{g}^{a}(\hat{x}), \psi_{g}^{a}(\hat{x}, u)\right) \in \mathbb{R}^{r}$, which is invertible with respect to $g$, and the remaining part $\left(\varphi_{g}^{b}(\hat{x}), \psi_{g}^{b}(\hat{x}, u)\right) \in \mathbb{R}^{n+m-r}$. The $r$ normalization equations

$$
\left(\varphi_{g}^{a}(\hat{x}), \psi_{g}^{a}(\hat{x}, u)\right)=c
$$

can then be solved into $g=\gamma(\hat{x}, u)$, and plugged into the remaining equations to yield the complete set of $n+m+p-r$ functionally independent invariants

$$
\begin{aligned}
I(\hat{x}, u) & :=\left(\varphi_{\gamma(\hat{x}, u)}^{b}(\hat{x}, u), \psi_{\gamma(\hat{x}, u)}^{b}(\hat{x}, u)\right) \\
J_{h}(\hat{x}, u, y) & :=\bar{\rho}_{\gamma(\hat{x}, u)}(y) .
\end{aligned}
$$

An invariant output error is then given by

$$
E(\hat{x}, u, y):=J_{h}(\hat{x}, u, h(\hat{x}, u))-J_{h}(\hat{x}, u, y) .
$$

Actually, every invariant output error $\tilde{E}$ must have the form

$$
\begin{aligned}
\tilde{E}(\hat{x}, u, y) & =\mathscr{F}\left(I(\hat{x}, u), J_{h}(\hat{x}, u, y)\right) \\
& =\mathscr{F}\left(I(\hat{x}, u), J_{h}(\hat{x}, u, h(\hat{x}, u))-E(\hat{x}, u, y)\right) \\
& =\mathscr{L}(I(\hat{x}, u), E(\hat{x}, u, y),)
\end{aligned}
$$

since $J_{h}(\hat{x}, u, h(\hat{x}, u))$, which is by construction invariant, must be a function of $I(\hat{x}, u)$.

Corollary 1: $\dot{\hat{x}}=F(\hat{x}, u, y)$ is a G-invariant pre-observer if and only if

$$
F(\hat{x}, u, y)=f(\hat{x}, u)+\sum_{i=1}^{n} \mathscr{L}_{i}(I(\hat{x}, u), E(\hat{x}, u, y)) w_{i}(\hat{x}),
$$

where the $\mathscr{L}_{i}$ 's are smooth functions such that for all $\hat{x}$ $\mathscr{L}_{i}(I(\hat{x}), 0)=0$, and the $w_{i}$ 's are point-wise independent invariant vector fields.

Notice once again the corollary says nothing about convergence.

Proof: We first follow [6], theorem 2.84, to build an invariant frame $w_{1}, \ldots, w_{n}$ : we apply once again the moving frame method, with $\Sigma:=\mathscr{X}$ and $\phi_{g}:=\varphi_{g}$. Locally, we can split $\varphi_{g}(x)$ into $\varphi_{g}^{a}(x) \in \mathbb{R}^{r}$, which is invertible with respect to $g$, and the remaining part $\varphi_{g}^{b}(x) \in \mathbb{R}^{n-r}$. The $r$ normalization equations $\varphi_{g}^{a}(x)=c$ can then be solved into $g=\gamma(x)$ (and when plugged into the remaining equations, yield a complete set of $n-r$ functionally independent invariants). The vector fields defined by

$$
w_{i}(x):=\left(D \varphi_{\gamma(x)}(x)\right)^{-1} \cdot \frac{\partial}{\partial x_{i}}, \quad i=1, \ldots, n,
$$

where $\left(\frac{\partial}{\partial x_{1}}, \ldots, \frac{\partial}{\partial x_{n}}\right)$ is the canonical frame, form the desired invariant frame. Indeed, they are clearly point-wise linearly independent. Each $w_{i}$ is invariant because for any group element $b$ and $x$ we have by construction

$$
\begin{aligned}
w_{i}\left(\varphi_{b}(x)\right) & =\left(D \varphi_{\gamma\left(\varphi_{b}(x)\right)}\left(\varphi_{b}(x)\right)\right)^{-1} \cdot \frac{\partial}{\partial x_{i}} \\
\left(D \varphi_{b}(x)\right)^{-1} \cdot w_{i}\left(\varphi_{b}(x)\right) & =\left[D \varphi_{\gamma\left(\varphi_{b}(x)\right)}\left(\varphi_{b}(x)\right) D \varphi_{b}(x)\right]^{-1} \cdot \frac{\partial}{\partial x_{i}}
\end{aligned}
$$

the group structure implies the two following equalities

$$
\begin{aligned}
\varphi_{\gamma\left(\varphi_{b}(x)\right)}\left(\varphi_{b}(x)\right) & =\varphi_{\gamma\left(\varphi_{b}(x)\right) \cdot b}(x) \\
D \varphi_{\gamma\left(\varphi_{b}(x)\right)}\left(\varphi_{b}(x)\right) D \varphi_{b}(x) & =D \varphi_{\gamma\left(\varphi_{b}(x)\right) \cdot b}(x)
\end{aligned}
$$

where - corresponds to the composition law on $G$. Since $\gamma_{\varphi_{b}(x) \cdot b} \equiv \gamma(x)$, we have

$$
\left(D \varphi_{b}(x)\right)^{-1} \cdot w_{i}\left(\varphi_{b}(x)\right)=\left(D \varphi_{\gamma(x)}(x)\right)^{-1} \cdot \frac{\partial}{\partial x_{i}}=w_{i}(x)
$$


We then go on with the proof of the corollary. The vector field $F$ in the corollary clearly is an pre-observer. Indeed,

$$
\begin{aligned}
F(x, u, h(x)) & =f(x, u)+\sum_{i=1}^{n} \mathscr{L}_{i}(I(\hat{x}), E(x, u, h(x))) w_{i}(\hat{x}) \\
& =f(x, u)+\sum_{i=1}^{n} \mathscr{L}_{i}(I(\hat{x}), 0) w_{i}(\hat{x})=f(x, u)
\end{aligned}
$$

By construction, it is invariant.

Conversely, assume $\dot{\hat{x}}=F(\hat{x}, u, y)$ is a G-invariant preobserver. It can be decomposed on the point-wise independent $w_{i}$ 's as

$$
F(\hat{x}, u, y)=\sum_{i=1}^{n} F_{i}(\hat{x}, u, y) w_{i}(\hat{x}),
$$

where the $F_{i}$ 's are smooth functions. Since it is an preobserver,

$$
f(x, u)=F(x, u, h(x, u))=\sum_{i=1}^{n} F_{i}(x, u, h(x, u)) w_{i}(x) .
$$

Since it is a G-invariant pre-observer and the $w_{i}$ 's are invariant,

$$
\begin{aligned}
& \sum_{i=1}^{n} F_{i}\left(\varphi_{g}(\hat{x}), \psi_{g}(\hat{x}, u), \rho_{g}(y)\right) w_{i}\left(\varphi_{g}(\hat{x})\right) \\
& \quad=D \varphi_{g}(\hat{x}) \cdot \sum_{i=1}^{n} F_{i}(\hat{x}, u, y) w_{i}(\hat{x}) \\
& \quad=\sum_{i=1}^{n} F_{i}(\hat{x}, u, y) w_{i}\left(\varphi_{g}(\hat{x})\right)
\end{aligned}
$$

hence

$$
F_{i}\left(\varphi_{g}(\hat{x}), \psi_{g}(\hat{x}, u), \rho_{g}(y)\right)=F_{i}(\hat{x}, u, y), \quad i=1, \ldots n .
$$

Therefore,

$$
\begin{aligned}
F(\hat{x}, u, y) & =f(\hat{x}, u)+[F(\hat{x}, u, y)-f(\hat{x}, u)] \\
& =f(\hat{x}, u)+\sum_{i=1}^{n}\left[F_{i}(\hat{x}, u, y)-F_{i}(\hat{x}, u, h(\hat{x}, u))\right] w_{i}(\hat{x}) .
\end{aligned}
$$

The functions $F_{i}(\hat{x}, u, y)-F_{i}(\hat{x}, u, h(\hat{x}, u))$ clearly are the components of an invariant output error; hence by theorem 1 , $F_{i}(\hat{x}, u, y)-F_{i}(\hat{x}, u, h(\hat{x}, u))=\mathscr{L}_{i}(I(\hat{x}, u), E(\hat{x}, u, y))$.

\section{DERIVE OUR OBSERVER FROM GENERAL THEORY}

We now show how the observer in section II can be derived from the theory developed in section III.

\section{A. The transformation group}

In this case $G$ is the 4-dimensional group $S^{1} \times S O(3)$. The map

$$
\phi_{(p, r)}\left(\begin{array}{c}
q \\
\vec{v} \\
\vec{a} \\
\vec{\omega}
\end{array}\right)=\left(\begin{array}{c}
p * q * r \\
r^{-1} * \vec{v} * r \\
r^{-1} * \vec{a} * r \\
r^{-1} * \vec{\omega} * r
\end{array}\right)
$$

defines a transformation group on the manifold $\mathscr{X} \times \mathscr{U}:=$ $\left(S O(3) \times \mathbb{R}^{3}\right) \times\left(\mathbb{R}^{3} \times \mathbb{R}^{3}\right)$.

\section{B. The invariant frame and errors}

The theory does not fully apply to the example developed in section 1, since it requires the group action to be free, which is the case if $\vec{v}$ and the gravity vector $\vec{G}$ are independent. Then the 6 invariant vector fields are

$$
\left(\begin{array}{c}
\hat{q} * \hat{\vec{v}} \\
0
\end{array}\right),\left(\begin{array}{c}
\hat{q} * \hat{\vec{G}} \\
0
\end{array}\right),\left(\begin{array}{c}
\hat{q} *(\hat{\vec{v}} \times \hat{\vec{G}}) \\
0
\end{array}\right)
$$

and

$$
\left(\begin{array}{c}
0 \\
\hat{\vec{v}}
\end{array}\right),\left(\begin{array}{c}
0 \\
\hat{\vec{G}}
\end{array}\right),\left(\begin{array}{c}
0 \\
\hat{\vec{v}} \times \hat{\vec{G}}
\end{array}\right) .
$$

A complete set of scalar invariant errors $\left(I^{\vec{v}}, I^{\vec{G}}, I^{\times}\right)$is simply obtained by the coordinates in the frame $(\hat{\vec{v}}, \hat{\vec{G}}, \hat{\vec{v}} \times \hat{\vec{G}})$ of $\hat{\vec{v}}-\vec{v}$ :

$$
\hat{\vec{v}}-\vec{v}=I^{\vec{v}} \hat{\vec{v}}+I^{\vec{G}} \hat{\vec{G}}+I^{\times} \hat{\vec{v}} \times \hat{\vec{G}} .
$$

We can construct an invariant observer with at least 18 independent gain.

\section{CONCLUSION}

We have proposed a systematic method to design preobservers preserving the symmetries of the original system under basic regularity condition relative to the action of symmetries-group on the state-space. We do not have up to now similar systematic procedures to tackle convergence and singularity of the group action on the state-space. Nevertheless, the velocity-aided inertial navigation problems illustrates that invariance can be a useful guide for the design of asymptotic nonlinear observers.

\section{REFERENCES}

[1] N. Aghannan and P. Rouchon. On invariant asymptotic observers. In Proceedings of the 41st IEEE Conference on Decision and Control, volume 2, pages 1479- 1484, 2002.

[2] N. Aghannan and P. Rouchon. An intrinsic observer for a class of lagrangian systems. IEEE AC, 48(6):936-945, 2003.

[3] F. Fagnani and J. Willems. Representations of symmetric linear dynamical systems. SIAM J. Control and Optim., 31:1267-1293, 1993.

[4] J.W. Grizzle and S.I. Marcus. The structure of nonlinear systems possessing symmetries. IEEE Trans. Automat. Control, 30:248-258, 1985.

[5] Ph. Martin, P. Rouchon, and J. Rudolph. Invariant tracking. ESAIM: Control, Optimisation and Calculus of Variations, 10:1-13, 2004.

[6] P. J. Olver. Equivalence, Invariants and Symmetry. Cambridge University Press, 1995.

[7] P. J. Olver. Classical Invariant Theory. Cambridge University Press, 1999.

[8] W. Respondek and I.A. Tall. Nonlinearizable single-input control systems do not admit stationary symmetries. Systems and Control Letters, 46:1-16, 2002.

[9] A.J. van der Schaft. Symmetries in optimal control. SIAM J. Control Optim., 25:245259, 1987.

[10] M.W. Spong and F. Bullo. Controlled symmetries and passive walking,. IEEE Trans. Automat. Control, 50:1025-1031, 2005. 